\title{
Perfiles motivacionales y diferencias en variables afectivas, motivacionales y de logro*
}

\author{
Motivational Profiles and Differences in Affective, \\ Motivational and Achievement Variables
}

Recibido: enero 22 de 2009 ～Revisado: marzo 4 de 2009 | Aceptado: marzo 18 de 2009

\author{
ANTONio Valle Arias*** \\ SUSANA RODRÍGUEZ \\ RAMÓN G. CABANACH \\ Universidad de A Coruña, España \\ José CARLOS NÚÑEZ PÉREZ *** \\ Julio ANTONIO GONZÁlez PIENDA \\ Universidad de Oviedo, España \\ PEDRo Rosario \\ Universidad de Minho, Portugal
}

Artículo de investigación, parte de otras investigaciones más amplias que han sido financiadas por la DGI-MEC (SEJ2006-01518) y por la DGU-MEC (EA2007-0242).

** Facultad de ciencias de la Educación, Universidad de La Coruña, Campus de Elviña s/n, 15071 A Coruña-España. Correos electrónicos: vallar@udc.es; susana@udc.es;rgc@udc.es

**** Facultad de Psicología, Universidad de Oviedo, Plaza Feijoo s/n, 33003 Oviedo-España.

Correos electrónicos: jcarlosn@uniovi.es; julioag@ uniovi.es

***** Instituto de Educación y Psicología. Universidade do Minho. Largo do Pazo, Braga-Portugal. Correo electrónico: prosario@iep.uminho.pt

\section{RESUMEN}

En este trabajo se pretende identificar si existen combinaciones de múltiples metas que dan lugar a diferentes perfiles motivacionales. La muestra está integrada por 1924 estudiantes universitarios. A través del análisis de conglomerados se han identificado seis perfiles motivacionales. Los resultados indican que el perfil motivacional que engloba a los estudiantes que están motivados para aprender, pero también para conseguir mejores resultados que los demás y para evitar dar una mala imagen ante ellos, son los que informan de un mejor rendimiento académico y los que creen también tener un nivel más alto de conocimientos en las materias académicas que están cursando. Por otro lado, los estudiantes con un perfil motivacional orientado al aprendizaje son los que hacen una valoración más alta de las tareas, los que tienen un mayor grado de control sobre su proceso de aprendizaje, y los que tienen unos niveles más bajos de ansiedad.

Palabras clave autores

Múltiples metas, perfiles motivacionales, metas académicas, estudiantes

universitarios.

Palabras clave descriptor

Motivación estudiantil, estudiantes universitarios, análisis cluster

\section{A B S T R ACT}

The purpose of this work is to identify whether there are combinations of multiple goals that lead to different motivational profiles. The sample is made up of 1924 university students. By means of cluster analysis, six motivational profiles were identified. The results indicate that the motivational profile that comprises students who are motivated to learn, but also to achieve better results that the rest and to avoid making a bad impression on them are the students who report better academic achievement and also the students who believe they have a higher level of knowledge in the academic subjects they are studying. However, students with a learning oriented motivational profile value the tasks more, have more control over their learning process, and have lower levels of anxiety.

Key words authors

Multiple Goals, Motivational Profiles, Academic Goals, University Students.

Key words plus

Student Motivation, College Students, Cluster Analysis. 
La teoría de las metas de logro se ha convertido en los últimos años en una de las líneas de investigación más importantes dentro de la motivación académica, tanto desde la perspectiva del alumno como del profesor (Brophy, 2005; Fernández-Arata, 2008). A la propuesta inicial de Dweck y Leggett (1988), que sólo diferenciaba entre metas de aprendizaje y metas de rendimiento, le siguió el enfoque tridimensional (Elliot, 1997, 1999; Elliot \& Harackiewicz, 1996; Middleton \& Midgley, 1997; Skaalvik, 1997), que incluía la diferenciación entre tendencias de aproximación y de evitación en las metas de rendimiento, y luego los modelos $2 \times 2$ (Elliot, 1999; Elliot \& McGregor, 2001; Pintrich, 2000a) que distinguían cuatro tipos de metas al contemplar las tendencias de aproximación y de evitación dentro de las metas de aprendizaje y de las metas de rendimiento, es decir, diferenciaban tanto la orientación "aprendizaje vs. rendimiento" como la dirección "aproximación vs. evitación" de las metas. De acuerdo con este último modelo, se pueden adoptar cuatro tipos de orientaciones de meta distintas: aproximación al dominio o al aprendizaje, evitación del dominio o del aprendizaje, aproximación al rendimiento, y evitación del rendimiento (Harackiewicz \& Linnenbrik, 2005).

Todo esto contribuyó a que la teoría de las metas de logro haya sufrido modificaciones importantes tanto respecto a su conceptualización como a los propios planteamientos de las investigaciones realizadas (Daniels, Haynes, Stupnisky, Perry, Newall \& Pekrun, 2008). Sin duda, una de las propuestas más relevantes y con mayor proyección en el propio contexto educativo, en el cual las metas tienen verdadero sentido y significado, es la de las múltiples metas. Ya el propio Pintrich (2003) estaba firmemente convencido de que muchas de las controversias en torno a las metas debían resolverse con más investigación empírica, contemplando como prioridades en la agenda de la investigación motivacional las siguientes: "La investigación futura sobre metas de logro necesita ir más allá de la diferenciación simplista entre metas de aprendizaje (buenas) vs. metas de rendimiento (malas) y considerar las múltiples metas, los múltiple resultados y los múltiples caminos para aprender y rendir en múltiples contextos" (p. 676).

La investigación sobre múltiples metas ha demostrado empíricamente que hay muchos estudiantes que, en lugar de adoptar una meta de manera exclusiva, optan por varias metas para implicarse en el aprendizaje. Así, ante situaciones en las que la actividad de aprendizaje es poco estimulante o interesante, razones distintas al interés intrínseco por la tarea podrían ser útiles para motivar su actuación. En tales casos, la posibilidad de optar por distintos motivos "obtener la aprobación de otros, conseguir premios y recompensas..." puede convertirse en un incentivo poderoso para promover y mantener el compromiso académico. Por tanto, el hecho de que los estudiantes opten por combinaciones de metas en situaciones escolares concretas suele ser una de las opciones que presenta mayores beneficios a nivel académico (Daniels et al., 2008; Ng, 2008; Pintrich, 2000b; Seifert, 1995; Suárez, Cabanach \& Valle, 2001; Valle, Cabanach, Núñez, González-Pienda, Rodríguez \& Piñeiro, 2003; Valle, Núñez, Cabanach, Rodríguez, González-Pienda \& Rosario, 2009; Wentzel, 2000).

Tomando como referencia algunos resultados encontrados en torno a la relación de las metas de rendimiento con los resultados académicos y a la relación de las metas de aprendizaje con el interés, Harackiewicz, Barron, Tauer, Carter y Elliot (2000) encontraron que cada tipo de meta (aprendizaje y rendimiento) estaba asociada con un indicador relevante de éxito (interés o rendimiento) pero no con el otro, lo que demostraba que aquellos estudiantes que adoptaran ambos tipos de metas eran, probablemente, los que conseguirían mejores resultados en ambas variables, es decir, un alto interés y un alto rendimiento. Por eso, como afirman Bouffard, Boisvert, Vezeau y Larouche (1995), la orientación motivacional más adecuada para un óptimo funcionamiento académico es aquella en la cual el estudiante no sólo está preocupado por el conocimiento y mejora de sus capacidades, sino también por conseguir un cierto nivel de rendimiento. Desde esta perspectiva, la orientación motivacional que mejor cumple esos dos requisitos es la de las múltiples metas, especialmente aquel 
perfil motivacional integrado por unas altas metas de aprendizaje acompañado de unas altas metas de aproximación al rendimiento.

La combinación de ambas orientaciones de meta implica la puesta en marcha de mecanismos de autorregulación del aprendizaje que permiten una utilización estratégica de las mismas, priorizando una u otra en cada situación particular, dependiendo de los propósitos, motivos o razones para implicarse o no en la tarea de aprendizaje. Esta forma de actuar supone una coordinación y un ajuste efectivo entre las razones personales y las demandas específicas del contexto de aprendizaje. El ejemplo propuesto por Paris, Byrnes \& Paris (2001) puede resultar esclarecedor respecto a esta idea. Pensemos en un estudiante que está realizando una actividad de matemáticas y que observa cómo otros compañeros terminan la tarea con gran rapidez. Si el estudiante se encuentra con dificultades para resolver la actividad, puede atribuir esta situación a su propia capacidad (en este caso, falta de capacidad para las matemáticas). Esta explicación podría conducir al estudiante a abandonar sus intentos de resolver con éxito la actividad y, en su lugar, copiar la respuesta de modo que ello le permita dar la imagen de que ha resuelto la tarea con prontitud, puesto que a ello va asociado el aparentar ante los demás que posee capacidad suficiente para resolver la tarea con facilidad. En este ejemplo hipotético el estudiante abandona sus objetivos iniciales de aprendizaje para adoptar metas de aproximación al rendimiento. Así, las condiciones que rodean la realización de las tareas pueden incidir en la forma de concebir la tarea y en el sentido de la propia competencia para resolverlas, lo que quedaría reflejado en la evolución o desarrollo de las orientaciones a meta adoptadas.

La metáfora de los "múltiples caminos" utilizada por Pintrich (2000b) supone un intento de sintetizar las divergencias existentes entre algunos de los resultados encontrados respecto a las ventajas e inconvenientes de adoptar unos tipos de metas u otros. Este autor plantea que las metas de aprendizaje y las metas de aproximación al rendimiento pueden adoptarse y fomentar diferentes patrones de motivación, afecto, uso de estrategias y rendi- miento a lo largo del tiempo. En este sentido, los estudiantes que adoptan distintas metas pueden seguir diversos caminos o trayectorias a lo largo del tiempo, con algunos de ellos finalizando en el mismo lugar en términos de rendimiento real, pero implicando una experiencia muy diferente hasta lograr el resultado final. Así, los estudiantes orientados al aprendizaje vivirían una experiencia "más tranquila y agradable" en términos de motivación, afecto positivo, esfuerzo y uso de estrategias, en su andadura hacia unos buenos resultados académicos. Por el contrario, aquellos estudiantes con metas de rendimiento, aun pudiendo alcanzar niveles equivalentes e incluso superiores a los estudiantes con metas de aprendizaje, pueden experimentar menos interés, afecto positivo y tal vez más ansiedad o afecto negativo, dadas sus preocupaciones por superar a los demás. Asimismo, cabe la posibilidad de que traten de demostrar un menor esfuerzo debido a su objetivo de parecer más capaces que sus compañeros y, en el caso de encontrarse con dificultades o fracasos a lo largo del camino, esto supondría costes para ellos en términos afectivos (menor interés y más afecto negativo), o los conduciría al empleo de estrategias orientadas a lograr su meta de ser mejores que los demás.

Bajo estos planteamientos, en este trabajo se plantean básicamente dos objetivos. En primer lugar, se pretende comprobar si existen combinaciones de múltiples metas que den lugar a diferentes perfiles motivacionales, los cuales podrían ser definidos en función del mayor o menor peso que tengan cada una de las metas dentro de cada perfil. Una vez hallados y definidos los perfiles motivacionales, en segundo lugar, se tratará de averiguar si existen diferencias significativas entre los grupos obtenidos respecto de algunas variables motivacionales relevantes para el aprendizaje: interés de las tareas, autoeficacia percibida, creencias de control, ansiedad ante los exámenes, satisfacción con los profesores, así como variables de rendimiento (rendimiento actual, expectativas de rendimiento y nivel percibido de conocimientos). Esta segunda parte del estudio constituye, en cierto sentido, la validación criterial de los perfiles encontrados y su utilidad a la hora de la planificación de las prácticas educativas. 


\section{Método}

\section{Participantes}

La muestra está integrada por 1924 estudiantes universitarios (17,6\% hombres y $82,4 \%$ mujeres) pertenecientes a dieciséis titulaciones de cinco universidades, geográficamente representativas, del territorio español. La selección de la muestra se ha realizado mediante un muestreo aleatorio estratificado por titulación. La selección del tamaño de los estratos se hizo por afijación proporcional al tamaño de cada titulación. Los participantes en la muestra final tienen edades comprendidas entre 18 y 46 años $(M=21,15)$. En cuanto a la distribución por cursos, el 32,7\% son de primero, el 34\% de segundo, el 20\% de tercero, el 9,5\% de cuarto, y el 3,5\% de quinto.

\section{Variables e Instrumentos}

- La evaluación de las metas académicas de los estudiantes se realizó mediante el Cuestionario de Metas Académicas propuesto por Skaalvik (1997). Este instrumento evalúa cuatro tipos de metas: metas de aprendizaje (o de aproximación a la tarea), metas de aproximación al rendimiento (o de mejora del yo), metas de evitación del rendimiento (o de defensa del yo) y metas de evitación del trabajo académico. Teniendo en cuenta que las metas de evitación del trabajo reflejan más bien un comportamiento de evitación y no un verdadero propósito o razón para implicarse o evitar implicarse a nivel académico, sólo se utilizaron los tres tipos de metas que la teoría e investigación motivacional ha contemplado como más representativas en contextos académicos. Este instrumento cumple los requisitos básicos en cuanto a su fiabilidad y validez. De hecho, ha sido adaptado para su uso en un importante número de trabajos con estudiantes universitarios (e.g. Rodríguez, Cabanach, Piñeiro, Valle, Núñez \& González-Pienda, 2001; Suárez, Cabanach \& Valle, 2001; Valle, Núñez, Cabanach, Rodríguez, González-Pienda, Rosário, Muñoz-Cadavid \& Cerezo, 2009).
- Para evaluar otras variables motivacionales como el valor de la tarea, las creencias de autoeficacia, las creencias de control y la ansiedad ante los exámenes se utilizó la parte motivacional del Motivated Strategies Learning Questionnaire, elaborado por Pintrich, Smith, García \& McKeachie (1991). Este instrumento ha sido adaptado en España por Roces (1996), para su aplicación con estudiantes universitarios, mostrando adecuadas características de fiabilidad y de validez, tanto estructural (de constructo) como predictiva.

- El resto de variables se evaluaron mediante una serie de ítems en los que se les solicitaba a los estudiantes información sobre su rendimiento actual, sus expectativas de rendimiento, su nivel de conocimientos y su grado de satisfacción con el profesor.

\section{Procedimiento}

Los datos relativos a las variables estudiadas fueron recogidos en cada uno de los centros universitarios de los que formaban parte los estudiantes que participaron en la investigación. Los cuestionarios fueron aplicados en un único momento temporal por personal especializado que colaboró en la investigación. A los participantes, que contestaron de forma individual y sin límite de tiempo a cada uno de los cuestionarios, se les recordaba que era muy importante que respondieran sinceramente a las distintas cuestiones planteadas.

\section{Análisis de datos}

Con el propósito de identificar los perfiles motivacionales de los estudiantes que participaron en la investigación se recurrió al análisis de conglomerados (método quick cluster analysis). Aunque dentro de las dos grandes categorías del análisis de conglomerados -métodos jerárquicos y no jerárquicos- los más utilizados para este tipo de estudios, son los primeros. La principal diferencia entre los métodos jerárquicos y no jerárquicos es que en estos últimos, el investigador debe especificar a priori los grupos 
que deben ser formados. En este caso se ha utilizado el quick cluster analysis, el cual además de ser un método no jerárquico también es un método de reasignación, es decir, permite que un individuo asignado a un grupo en un determinado paso del proceso, sea reasignado a otro grupo en un paso posterior, si esto optimiza el criterio de selección. Este análisis de conglomerados no sólo es el procedimiento más adecuado para establecer perfiles en una muestra amplia de sujetos (Hair, Anderson, Tatham \& Black, 1998), sino que es una de las soluciones más recomendadas para la identificación de múltiples metas (Pastor, Barron, Davis \& Miller, 2004).

Los perfiles motivacionales se han definido a partir de las diferentes combinaciones de tres tipos de metas "metas de aprendizaje, metas de aproximación al rendimiento y metas de evitación del rendimiento" que evalúa el Cuestionario de Metas Académicas propuesto por Skaalvik (1997). Para eliminar el efecto debido a las diferencias en la medida de las metas (debido a que el número de ítems de cada una de las subescalas de metas no es el mismo), se ha realizado el análisis de conglomerados después de haber estandarizado las puntuaciones directas.

El criterio seguido en la elección del número de conglomerados fue el maximizar las diferencias inter-clusters con el fin de conseguir establecer el mayor número posible de grupos con diferentes combinaciones de metas. Además, a este criterio se le añadió también la viabilidad teórica y significación psicológica de cada uno de los grupos que representaban los distintos perfiles motivacionales.

Por último, una vez identificados los perfiles motivacionales, se analizó la significación estadística de las diferencias existentes entre los grupos en la percepción del rendimiento académico, nivel de conocimientos y satisfacción con el profesor, y también en cuanto a algunas variables motivacionales y a su interés académico. Dado que las variables incluidas en este estudio se encuentran correlacionadas significativamente (véase Tabla 1 ), se ha optado por llevar a cabo un análisis multivariado de la varianza (MANOVA). Puesto que el factor está

TABLA 1

Correlaciones, medias, desviaciones típicas de las variables objeto de estudio

\begin{tabular}{lcccccccccccc}
\hline & 1 & 2 & 3 & 4 & 5 & 6 & 7 & 8 & 9 & 10 & Media & D. Típica \\
\hline 1. REN.ACT. & - & & & & & & & & & & 3,64 & 0,63 \\
2. EXP.REN. & $0,24^{* * *}$ & - & & & & & & & & & 2,47 & 0,68 \\
3. CONOC. & $0,22^{* *}$ & $0,42^{*}$ & - & & & & & & & & 3,17 & 0,79 \\
4. SAT.PROF. & $0,06^{* *}$ & $0,29^{* * *}$ & $0,47^{* *}$ & - & & & & & & & 3,47 & 1,06 \\
5. VAL.TAR. & 0,04 & $0,21^{* * *}$ & $0,41^{* *}$ & $0,58^{* * *}$ & - & & & & & & 3,68 & 0,96 \\
6. CRE.CONT. & $-0,04$ & $0,25^{* *}$ & $0,35^{* *}$ & $0,54^{* *}$ & $0,53^{* *}$ & - & & & & & 3,65 & 0,79 \\
7. CRE.AUT. & $0,15^{* *}$ & $0,56^{* *}$ & $0,52^{* *}$ & $0,50^{* *}$ & $0,53^{* *}$ & $0,64^{* *}$ & - & & & & 3,38 & 0,76 \\
8. ANS.EX. & $-0,12^{* *}$ & $-0,32^{* *}$ & $-0,14^{* *}$ & $-0,12^{* * *}$ & $-0,04$ & $-0,14^{* *}$ & $-0,37^{* *}$ & - & & & 3,04 & 0,87 \\
9. M.APR. & $0,10^{* *}$ & $0,20^{* * *}$ & $0,37^{* *}$ & $0,47^{* *}$ & $0,80^{* *}$ & $0,44^{* *}$ & $0,50^{* *}$ & $-0,00$ & - & & 3,61 & 0,83 \\
10. M.A.R. & $0,07^{* * *}$ & $0,18^{* *}$ & $0,16^{* *}$ & $0,08^{* *}$ & $0,08^{* *}$ & $0,07^{* *}$ & $0,20^{* *}$ & $0,08^{* *}$ & $0,16^{* *}$ & - & 2,00 & 0,88 \\
11. M.E.R. & $-0,02$ & $-0,03$ & $-0,03$ & $-0,01$ & $-0,02$ & $-0,00$ & $-0,08^{* *}$ & $0,32^{* * *}$ & 0,01 & $0,30^{* *}$ & 2,32 & 1,04 \\
\hline
\end{tabular}

REN.ACT. $=$ rendimiento actual, EXPREN.$=$ expectativas de rendimiento, CONOC.$=$ nivel de conocimientos, SAT.PROF. $=$ satisfacción con el profesor, VAL.TAR. $=$ valor de la tarea, CRE.CONT. $=$ creencias de control, CRE.AUT. $=$ creencias de autoeficacia, ANS.EX. = ansiedad ante los exámenes, M.APR. = metas de aprendizaje, M.A.R. = metas de aproximación al rendimiento, M.E.R. = metas de evitación del rendimiento.

$* \mathrm{p}<.05, * * \mathrm{p}<.01$

Fuente: elaboración propia. 
integrado por más de dos niveles o grupos se utilizó como prueba de comparaciones múltiples post-hoc la prueba de Scheffé. Todos los análisis estadísticos se han realizado con el programa SPSS 16.0.

\section{Resultados}

\section{Identificación de Perfiles Motivacionales}

Los resultados del análisis de conglomerados permitieron diferenciar seis grupos caracterizados por distintas combinaciones de metas y que dieron lugar a otros tantos perfiles motivacionales (véase Figura 1).

Un primer grupo, integrado por 305 estudiantes (15,9\% de los participantes), está caracterizado por puntuaciones bajas en todas las metas evaluadas. Este grupo se ha definido por un perfil con baja motivación generalizada (Grupo BMG).

El segundo grupo, formado por 192 estudiantes (10\% de los participantes), se caracteriza por un predominio de metas de evitación del rendimiento (Grupo MER). Este grupo se ha definido por un

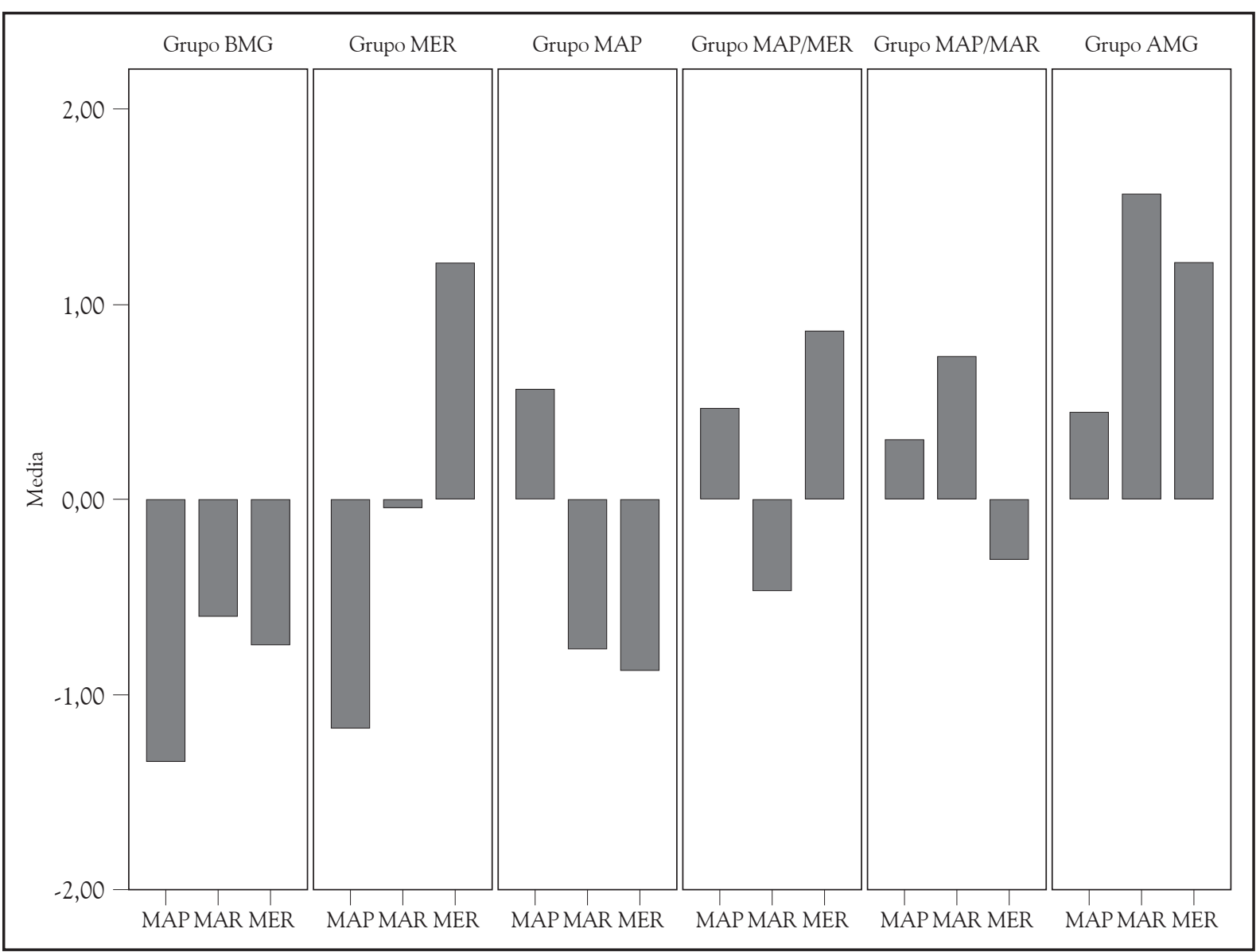

FIGURA 1

Representación gráfica de los perfiles motivacionales identificados a través del análisis de conglomerados.

MAP: metas de aprendizaje; MAR: metas de aproximación al rendimiento; MER: metas de evitación del rendimiento. Grupo BMG (baja motivación generalizada), Grupo MER (predominio de metas de evitación del rendimiento), Grupo MAP (predominio de metas de aprendizaje), Grupo MAP/MER (predominio de metas de aprendizaje y de metas de evitación del rendimiento), Grupo MAP/MAR (predominio de metas de aprendizaje y de metas de aproximación al rendimiento), Grupo AMG (alta motivación generalizada).

Fuente: elaboración propia. 
perfil motivacional orientado a evitar una mala imagen ante los demás.

El tercer grupo, integrado por 457 estudiantes (23,8\% de los participantes), se caracteriza por un predominio de metas de aprendizaje (Grupo MAP). Este grupo se ha definido por un perfil motivacional orientado al aprendizaje.

El cuarto grupo, formado por 302 estudiantes $(15,7 \%$ de los participantes), está caracterizado por un predominio de metas de aprendizaje y de metas de evitación del rendimiento (Grupo MAP/MER). Este grupo se ha definido por un perfil motivacional orientado al aprendizaje y a evitar una mala imagen ante los demás.

El quinto grupo, integrado por 444 estudiantes (23,1\% de los participantes), se caracteriza por un predominio de metas de aprendizaje y de metas de aproximación al rendimiento (Grupo MAP/MAR). Este grupo se ha definido por un perfil motivacional orientado al aprendizaje y a conseguir mejores resultados académicos que los demás.

Finalmente, el sexto grupo, formado por 224 estudiantes (11,6\% de los participantes) está caracterizado por puntuaciones altas en todas las metas evaluadas. Este grupo se ha definido por un perfil con alta motivación generalizada (Grupo AMG).

\section{Diferencias intergrupo en variables motivacionales, afectivas y de logro}

Por lo que se refiere a las diferencias entre los grupos en variables motivacionales, afectivas y de logro, los resultados derivados del MANOVA indican que la nueva variable creada (tipos de perfil motivacional) explica una cantidad de varianza estadísticamente significativa $\left(\lambda_{\text {Wilks }}=.525, F(40\right.$, $\left.8332)=33,219, p=.000, \eta^{2}=.121\right)$, lo que nos informa de la validez de los perfiles identificados; es decir, un $12,1 \%$ de la variabilidad observada en el conjunto de variables motivacionales, afectivas y de logro es explicado por la pertenencia a alguno de los seis tipos de perfiles motivacionales hallados.

Pero, más allá de estas consideraciones generales, así mismo, dichos perfiles muestran capacidad predictiva para todas las variables analizadas (véase Tabla 2). Según se observa en esta tabla, el valor de la tarea se encuentra explicado ampliamente por la variable perfil motivacional (en un 39,2\%), seguido por la creencias de autoeficacia (un 16\%), satisfacción con el profesor (12,9\%), las creencias de control (un 11,1\%), la percepción de conocimientos adquiridos (un 9\%), la ansiedad ante los exámenes (un 6,9\%), las notas finales esperadas (un 4,5) y, por último, el rendimiento actual (un $1,3 \%)$.

Más concretamente, los resultados referidos al rendimiento académico percibido y al nivel de conocimientos indican que el grupo con un perfil motivacional orientado a evitar una mala imagen ante los demás, (Grupo MER), es el que presenta unas puntuaciones más bajas que el resto de los grupo, aunque sí es verdad que esas diferencias no son estadísticamente significativas con respecto a todos los grupos. De hecho, en muchos casos, esas puntuaciones más bajas en estas variables son compartidas con el grupo con un perfil de baja motivación generalizada (Grupo BMG). Por otro lado, las puntuaciones más altas en rendimiento académico y nivel de conocimientos son las del grupo con un perfil de alta motivación generalizada (Grupo AMG), aunque también en este caso, algunas de esas puntuaciones más altas son compartidas también con otros grupos (véanse Tabla 2 y Figura 2).

En cuanto al grado de satisfacción que tienen los estudiantes con sus profesores, los resultados indican que el grupo con un perfil motivacional orientado al aprendizaje (Grupo MAP) es el que presenta las puntuaciones más altas en esta variable, mientras que el grupo con baja motivación generalizada (Grupo BMG) es el que presenta las puntuaciones más bajas (véanse Tabla 2 y Figura 2).

Por lo que se refiere a algunas variables motivacionales contempladas en este trabajo, los resultados indican que el grupo con un perfil motivacional orientado al aprendizaje (Grupo MAP) es el que muestra unas puntuaciones más altas en el valor asignado a las tareas, en creencias de control y también en creencias de autoeficacia, aunque en este último caso esas puntuaciones son ligeramente superiores en el grupo con un perfil motivacional orientado al aprendizaje y a conseguir mejores resultados que los demás (Grupo MAP/MAR) y en 
TABLA 2

Medias, desviaciones típicas y significación estadística de las diferencias entre los perfiles motivacionales en algunas variables relevantes para el aprendizaje

\begin{tabular}{|c|c|c|c|c|c|c|c|c|c|c|c|c|c|}
\hline & \multicolumn{2}{|c|}{$\begin{array}{c}\text { Grupo } \\
\text { BMG } \\
{[1]}\end{array}$} & \multicolumn{2}{|c|}{$\begin{array}{c}\text { Grupo } \\
\text { MER } \\
\text { [2] }\end{array}$} & \multicolumn{2}{|c|}{$\begin{array}{c}\text { Grupo } \\
\text { MAP } \\
\text { [3] }\end{array}$} & \multicolumn{2}{|c|}{$\begin{array}{c}\text { Grupo } \\
\text { MAP/MER } \\
\text { [4] }\end{array}$} & \multicolumn{2}{|c|}{$\begin{array}{c}\text { Grupo } \\
\text { MAP/MAR } \\
\text { [5] }\end{array}$} & \multicolumn{2}{|c|}{$\begin{array}{c}\text { Grupo } \\
\text { AMG } \\
\text { [6] }\end{array}$} & \multirow[t]{2}{*}{$F_{(5,1918)} /\left(\eta^{2}\right)$} \\
\hline & M & DT & M & DT & M & DT & M & DT & M & DT & M & DT & \\
\hline end. A & 3,54 & 0,68 & 3,49 & 0,64 & 3,67 & 0,59 & 3,65 & 0,61 & 3,67 & 0,64 & 3,74 & 0,58 & $5,16^{* * * *} /(.013)$ \\
\hline xpect. Rend. & 2,28 & 0,67 & 2,20 & 0,68 & 2,49 & 0,66 & 2,46 & 0,63 & 2,62 & 0,66 & 2,62 & 0,74 & $18,19^{* * * *} /(.045)$ \\
\hline ivel Conocim & 2,80 & 0,87 & 2,74 & 0,80 & 3,32 & 0,70 & 3,17 & 0,69 & 3,32 & 0,74 & 3,43 & 0,79 & $37,95^{* * * *} /(.090)$ \\
\hline atisf. con Prof. & 2,75 & 1,11 & 2,98 & 1,11 & 3,75 & 0,93 & 3,71 & 0,96 & 3,68 & 0,96 & 3,60 & 0,96 & \\
\hline Glor Tarea & 2,61 & 0,84 & 2,75 & 0,82 & 4,18 & 0,70 & 4,04 & 0,67 & 3,94 & 0,73 & 3,89 & 0,78 & $247,31^{* * *} /(.392)$ \\
\hline Crecti. & 3,15 & 0,92 & 3,29 & 0,84 & 3,85 & 0,72 & 3,80 & 0,68 & 3,78 & 0,62 & 3,74 & 0,75 & $47,98^{* * * *} /(.111)$ \\
\hline reen Autoefic & 2,92 & 0,78 & 2,80 & 0,78 & 3,57 & 0,69 & 3,41 & 0,68 & 3,61 & 0,61 & 3,59 & 0,70 & $72,87^{* * * *} /(.160)$ \\
\hline Ansied. Exámenes & 2,94 & 0,89 & 3,33 & 0,74 & 2,76 & 0,88 & 3,32 & 0,90 & 2,95 & 0,76 & 3,33 & 0,78 & $28,57^{* * * *} /(.069)$ \\
\hline
\end{tabular}

$* \mathrm{p}<.05, * * \mathrm{p}<.01, * * * \mathrm{p}<.001$

$\eta^{2}=$ Tamaño del efecto. Grupo BMG: Baja motivación generalizada; Grupo MER: Predominio de metas de evitación del rendimiento; Grupo MAP: Predominio de metas de aprendizaje; Grupo MAP/MER: Predominio de metas de aprendizaje y de metas de evitación del rendimiento; Grupo MAP/MAR: Predominio de metas de aprendizaje y de metas de aproximación al rendimiento; Grupo AMG: Alta motivación generalizada.

Fuente: elaboración propia.

el grupo con alta motivación generalizada (Grupo $\mathrm{AMG})$. Por el contrario, en la mayor parte de estas variables, tanto el grupo con un perfil de baja motivación generalizada (Grupo BMG) como el grupo con un perfil motivacional orientado a evitar una mala imagen ante los demás (Grupo MER) son los que presentan unas puntuaciones más bajas que el resto de los grupos (véanse Tabla 2 y Figura 2).

Sin embargo, los niveles más altos de ansiedad ante los exámenes son los obtenidos por el Grupo MER, el Grupo MAP/MER y el Grupo AMG, es decir, todos aquellos perfiles motivacionales donde de modo individual o combinado tienen presencia importante las metas de evitación del rendimiento. Por otro lado, los niveles más bajos en ansiedad son los del grupo con un perfil motivacional orientado al aprendizaje (Grupo MAP) (véanse Tabla 2 y Figura 2).

Todas las difererencias son estadísticamente significativas excepto entre los siguientes grupos(prueba de Scheffé): Rend. Actual: 1-2,
$1-3,1-4,1-5,2-3,2-4,2-5,3-4,3-5,3-6,4-5,4-6$, 5-6; Expect. Rend.: 1-2, 1-4, 3-4, 3-5, 3-6, 4-5, 4-6, 5-6; Nivel Conocim.: 1-2, 3-4, 3-5, 3-6, 4-5, 5-6; Satisf. con Prof.: 1-2, 3-4, 3-5, 3-6, 4-5, 4-6, 5-6; Valor Tarea: 1-2, 3-4, 4-5, 4-6, 5-6; Creen. Control: 1-2, 3-4, 3-5, 3-6, 4-5, 4-6, 5-6; Creen. Autoefic.: 1-2,. 3-4, 3-5, 3-6, 4-6; Ansied. Exámenes: 1-3, $1-5,2-4,2-6,4-6$.

\section{Discusión}

Aunque algunos teóricos de la motivación pronosticaban hace unos años que el estudio de los perfiles motivacionales se iba a convertir en uno de los temas de investigación prioritarios, lo cierto es que el número e impacto de estos trabajos ha sido menor de lo esperado. Es más, la perspectiva de las múltiples metas, cuyo argumento central reside en la combinación de varias metas y en la definición de los perfiles motivacionales derivados 


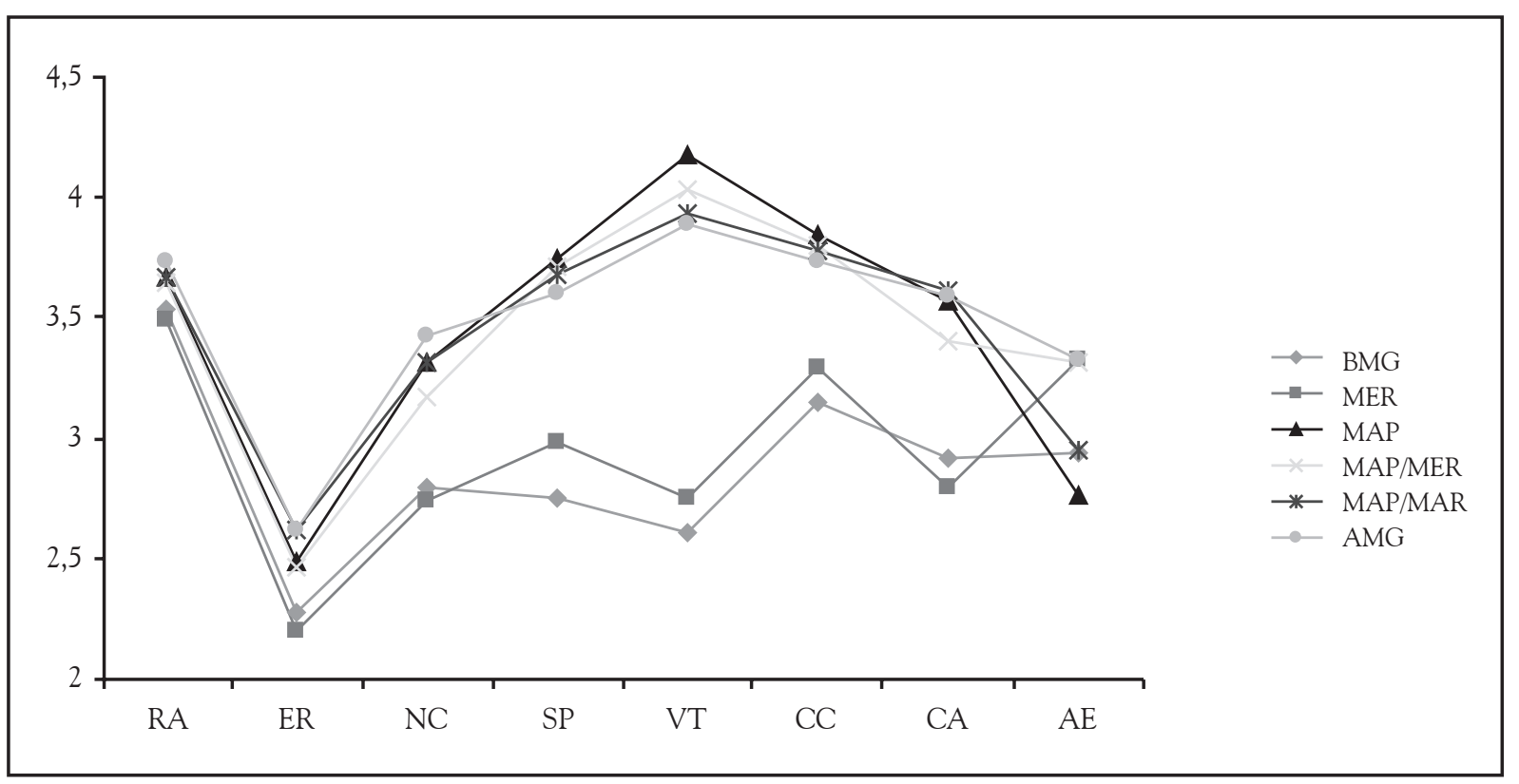

Figura 2

Representación gráfica del nivel de cada perfil motivacional en las diferentes variables medidas.

RA (rendimiento actual), ER (expectativas de rendimiento futuro), NC (nivel de conocimiento percibido), SP (satisfacción con profesor), VT (valor de las tareas), CC (creencias de control), CA (creencias de autoeficacia), AE (ansiedad ante los exámenes). BMG (baja motivación generalizada), MER (predominio de metas de evitación del rendimiento), MAP (predominio de metas de aprendizaje), MAP/MER (predominio de metas de aprendizaje y de metas de evitación del rendimiento), MAP/MAR (predominio de metas de aprendizaje y de metas de aproximación al rendimiento), AMG (alta motivación generalizada).

Fuente: elaboración propia.

de esas combinaciones, es una línea de trabajo de enorme proyección y con grandes posibilidades de futuro, pero todavía sigue ocupando una parte muy pequeña dentro de la investigación motivacional en contextos educativos.

Con este trabajo se pretende contribuir a desarrollar una línea de investigación que precisa de un mayor número de aportaciones centradas en analizar la dinámica de las múltiples metas y, sobre todo, en comprender determinados perfiles motivacionales, que hasta hace pocos años eran difícilmente explicables por la investigación motivacional. Se trata de aquellos perfiles donde predominan tipos de metas tradicionalmente consideradas antagónicas e incompatibles dentro de la misma persona.

El principal propósito de este trabajo fue analizar las distintas combinaciones de metas y luego definir los perfiles motivacionales, en una muestra de estudiantes universitarios. Los resultados demostraron tanto la existencia de perfiles motivacio- nales en los que predomina una única meta, como de perfiles motivacionales en los que predominan varias metas. Es más, cada uno de los grupos identificados presentan patrones diferentes de aprendizaje dependiendo de las metas dominantes en sus perfiles motivacionales $(\mathrm{Ng}, 2008)$.

Mediante el análisis de conglomerados se identificaron seis perfiles motivacionales distintos. Un primer grupo con un perfil de baja motivación generalizada (Grupo BMG). Un segundo grupo con un perfil motivacional orientado a evitar una mala imagen ante los demás (Grupo MER). Un tercer grupo con un perfil motivacional orientado al aprendizaje (Grupo MAP). Un cuarto grupo con un perfil motivacional orientado al aprendizaje y a evitar una mala imagen ante los demás (Grupo MAP/MER). Un quinto grupo con un perfil motivacional orientado al aprendizaje y a conseguir mejores resultados académicos que los demás (Grupo MAP/MAR). Por último, un sexto 
grupo con un perfil de alta motivación generalizada (Grupo AMG).

Con respecto a las diferencias entre los grupos en variables de logro, los resultados indicaron que el perfil motivacional donde predominan las tres metas evaluadas (Grupo AMG), es el que presenta unas puntuaciones más altas en cuanto al rendimiento académico percibido y al nivel de conocimientos. Es decir, aquellos estudiantes que están motivados para aprender, pero también para conseguir mejores resultados que los demás y para evitar una mala imagen ante ellos, son los que informan de un mejor rendimiento académico y los que creen también tener un nivel más alto de conocimientos en las materias académicas que están cursando. Por tanto, parece evidente que adoptar múltiples metas simultáneamente es una de las claves para el aprendizaje exitoso en el ámbito universitario (Barron \& Harackiewicz, 2000, 2001).

Por el contrario, el perfil motivacional centrado en evitar una mala imagen ante los demás (Grupo MER) es el que obtiene las puntuaciones más bajas en estas variables. El temor al fracaso, que caracteriza a los estudiantes con este perfil motivacional, los lleva a una constante preocupación por evitar parecer incompetentes ante los demás. Esto, que puede tener sus beneficios para la propia imagen, suele tener consecuencias muy negativas para la implicación y el compromiso del estudiante a nivel académico. De hecho, muchas de las conductas en las que se implican estos estudiantes son antagónicas con un aprendizaje exitoso (Gabriele, 2007).

En cuanto a las variables motivacionales contempladas en este trabajo, los resultados indican que los estudiantes con un perfil motivacional orientado al aprendizaje (Grupo MAP) son los que hacen una valoración más alta de las tareas y los que tienen un mayor grado de control sobre su proceso de aprendizaje. Además, junto con otros grupos, son los que tienen unas creencias de autoeficacia más altas. Por otro lado, tanto el grupo con baja motivación generalizada (Grupo BMG) como el grupo con un perfil orientado a evitar una mala imagen ante los demás (Grupo MER), son los que obtienen las puntuaciones más bajas en estas variables. Estos resultados coinciden con varios trabajos en los que las metas de aprendizaje se asociaron con creencias más altas sobre el valor de la tarea (e.g., Ames, 1992; Harackiewicz, Barron \& Elliot, 1998; Wolters, Yu \& Pintrich, 1998) y también con mayores niveles de autoeficacia (e.g., Bandalos, Finney \& Geske, 2003; Middleton \& Midgley, 1997; Skaalvik, 1997).

El perfil motivacional orientado al aprendizaje (Grupo MAP) es el que presenta los niveles más bajos de ansiedad, mientras que todos aquellos grupos en los que hay una dominancia -individual o combinada con otras metas- de metas de evitación del rendimiento, son los que tienen los niveles más altos de ansiedad. También aquí los resultados coinciden, en general, con las aportaciones de otros estudios en los que se encontró una relación positiva entre metas de evitación del rendimiento y ansiedad (e.g., Middleton \& Midgley, 1997) y en la que los estudiantes con metas de aprendizaje eran los que experimentaban un menor nivel de ansiedad ante los exámenes (e.g., Pintrich, 2000b).

\section{Implicaciones educativas}

Aunque los datos aportados por este estudio tienen diferentes y claras aportaciones para la práctica educativa, aquí vamos a destacar sólo dos de las más interesantes.

En primer lugar, los datos de nuestro estudio muestran que los perfiles más desadaptativos son los que muestran un nivel bajo en metas de aprendizaje (BMG y MER), mientras que aquellos con un nivel aceptable o alto de metas de aprendizaje conllevan un patrón adaptativo a nivel cognitivomotivacional, afectivo y de logro (MAP, MAP/ MER, MAP/MAR y AMG). En este sentido, se podría asumir que la presencia en el perfil motivacional de un componente de metas de aprendizaje, supondría un factor de protección en la medida que lleva al estudiante a valorar en mayor medida las tareas académicas, cree más en su capacidad para afrontar los aprendizajes escolares, muestra mayores creencias de control personal sobre los resultados de su trabajo, percibe el contexto de un 
modo más positivo, etc. Al contrario, la ausencia de metas de aprendizaje en el perfil motivacional implicaría un factor de riesgo serio.

En segundo lugar, por tanto, parece relevante identificar el tipo de perfil motivacional del estudiante con el fin de ajustar la estrategia docente a las necesidades motivacionales del alumno y evitar, en el caso de los estudiantes en situación de riesgo, el fracaso en el aprendizaje y el abandono escolar. En este sentido, y dado que el componente motivacional es esencial para el trabajo cognitivo, los docentes deberían disponer de información relativa al perfil motivacional de sus alumnos con el fin de ajustar su trabajo a las necesidades motivacionales de los estudiantes y, así, trabajar dentro de lo que podríamos denominar zona de desarrollo próximo motivacional. Se trata de asumir que para que un alumno avance de un estado motivacional complejo y de riesgo, hacia otro complejo pero adaptativo, es imprescindible partir del estado motivacional actual. Por tanto, motivar a los alumnos para el trabajo académico implica, en cierto sentido, adaptarse a los intereses de los alumnos pero no para quedarse ahí (lo cual en muchos casos llevaría a resultados no deseados), sino para poder mejorar o reorientar ese perfil motivacional actual hacia otro, con un claro componente de aprendizaje. En este sentido, Brophy (2004) señala que aunque los estudiantes entren en clase con un tipo u otro de perfil motivacional (orientados hacia una u otra meta académica), dicho perfil puede ser modificado como consecuencia de la participación del estudiante en contextos de aprendizaje apropiados.

\section{Comentarios finales}

Con independencia de las aportaciones de este trabajo y de otros similares, todavía siguen existiendo muchas dudas en torno a la dinámica de las múltiples metas. Una de ellas tiene que ver con su funcionamiento ante una tarea concreta, es decir, saber si los estudiantes combinan esas metas de manera simultánea o si, por el contrario, hay una alternancia en ellas (Harackiewicz \& Linnenbrik, 2005). Por eso, la investigación motivacional debe ayudar a que se aclaren todas estas dudas, y así contribuir a que la perspectiva de las múltiples metas ocupe definitivamente la posición destacada que los teóricos de la motivación le asignaban hace unos años. Para que esto ocurra, también será preciso que "nuestros programas y métodos de investigación sean mucho más sofisticados y complejos para captar estas dinámicas, incluyendo tanto estudios microgenéticos como longitudinales con múltiples resultados en múltiples contextos" (Pintrich, 2003, p. 677).

\section{Referencias}

Ames, C. (1992). Classrooms: Goals, structures and student motivation. Journal of Educational Psycho$\log y, 84,261-271$.

Bandalos, D., Finney, S. \& Geske, J. (2003). A model of statistics performance based on achievement goal theory. Journal of Educational Psychology, 95, 604-616.

Barron, K. E. \& Harackiewicz, J. M. (2000). Achievement goals and optimal motivation: A multiple goals approach. En C. Sansone \& J. M. Harackiewicz (Eds.), Intrinsic and extrinsic motivation: The search for optimal motivation and performance (pp. 229-254). New York: Academic Press.

Barron, K. E. \& Harackiewicz, J. M. (2001). Achievement goals and optimal motivation: Testing multiple goal models. Journal of Educational Psychology, 80, 706-722.

Bouffard, T., Boisvert, J., Vezeau, C. \& Larouche, C. (1995). The impact of goal orientation on selfregulation and performance among college students. British Journal of Educational Psychology, 65, 317-329.

Brophy, J. (2004). Motivating students to learn. Mahwah, New Jersey: LEA.

Brophy, J. (2005). Goal theorists should move on from performance goals. Educational Psychologist, 40(3), 167-176.

Daniels, L. M., Haynes, T. L., Stupnisky, R. H., Perry, R. P., Newall, N. E. \& Pekrun, R. (2008). Individual differences in achievement goals: A longitudinal study of cognitive, emotional, and achievement 
outcomes. Contemporary Educational Psychology, 33, 584-608.

Dweck, C. S. \& Leggett, E. L. (1988). A social-cognitive approach to motivation and personality. Psychology Review, 95, 256-273.

Elliot, A. (1997). Integrating the "classic" and "contemporary" approaches to achievement motivation: A hierarchical model of approach and avoidance achievement motivation. En M. L. Maehr \& P. R. Pintrich (Eds.), Advances in motivation and achievement (pp. 143-179). Greenwich, CT: JAI.

Elliot, A. J. (1999). Approach and avoidance motivation and achievement goals. Educational Psychologist, 34, 169-189.

Elliot, A. \& Harackiewicz, J. M. (1996). Approach and avoidance achievement goals and intrinsic motivation: A mediational analysis. Journal of Personality and Social Psychology, 70, 461-465.

Elliot, A. J. \& McGregor, H. A. (2001). A 2x2 achievement goal framework. Journal of Personality and Social Psychology, 80, 501-519.

Fernández-Arata, J. M. (2008). Desempeño docente y su relación con orientación a la meta, estrategias de aprendizaje y autoeficacia: un estudio con maestros de primaria de Lima, Perú. Universitas Psychologica, 7, 385-401.

Gabriele, A. J. (2007). The influence of achievement goals on the constructive activity of low achievers during collaborative problem solving. British Journal of Educational Psychology, 77, 121-141.

Hair, J. F., Anderson, R. E., Tatham, R. L. \& Black, W. C. (1998). Multivariate data analysis ( $5^{\text {th }}$ ed.). Upper Saddle River, NJ: Prentice-Hall.

Harackiewicz, J. M., Barron, K. E. \& Elliot, A. (1998). Rethinking achievement goals: When are they adaptative for college students and why? Educational Psychologist, 33, 1-21.

Harackiewicz, J. M., Barron, K. E., Tauer, J. M., Carter, S. M. \& Elliot, A. J. (2000). Short-term and longterm consequences of achievement goals: Predicting interest and performance over time. Journal of Educational Psychology, 92, 316- 330.

Harackiewicz, J. M. \& Linnenbrik, E. A. (2005). Multiple achievement goals and multiple pathways for learning: The agenda and impact of Paul R. Pintrich. Educational Psychologist, 40, 75-84.
Middleton, M. \& Midgley, C. (1997). Avoiding the demonstration of lack of ability: An unexplored aspect of goal theory. Journal of Educational Psychology, 89, 710- 718.

$\mathrm{Ng}, \mathrm{C} . \mathrm{H}$. (2008). Multiple-goal learners and their differential patterns of learning. Educational Psychology, 28, 439-456.

Paris, S. G., Byrnes, J. P. \& Paris, A. H. (2001). Constructing theories, identities, and actions of selfregulated learners. En B. J. Zimmerman \& D. H. Schunk (Eds.), Self-regulated learning and academic achievement: Theoretical perspectives ( $2^{\text {nd }} \mathrm{ed}$., pp. 253-287). Mahwah, NJ: Lawrence Erlbaum.

Pastor, D., Barron, K. E., Davis, S. L. \& Miller, B. J. (2004, April). College students' achievement goal orientation profiles. Paper presented at the meeting of the American Educational Research Association, San Diego, CA.

Pintrich, P. R. (2000a). An achievement goal theory perspective on issues in motivation terminology, theory, and research. Contemporary of Educational Psychology, 25, 92-104.

Pintrich, P. R. (2000b). Multiple goals, multiple pathways: The role of goal orientation in learning and achievement. Journal of Educational Psycho$\log y, 92,544-555$.

Pintrich, P. R. (2003). A motivational science perspective on the role of student motivation in learning and teaching contexts. Journal of Educational Psychology, 95, 667-686.

Pintrich, P. R., Smith, D. A., Garcia, T. \& McKeachie, W. J. (1991). A manual for the use of the Motivated Strategies for Learning Questionnaire (MSLQ). Ann Arbor, MI: National Center for Research to Improve Postsecundary Teaching and Learning.

Roces, C. (1996). Estrategias de aprendizaje y motivación en la universidad. Tesis Doctoral no publicada, Universidad de Navarra, España.

Rodríguez, S., Cabanach, R. G., Piñeiro, I., Valle, A., Núñez, J. C. \& González-Pienda, J. A. (2001). Metas de aproximación, metas de evitación y múltiples metas académicas. Psicothema, 13, 546-550.

Seifert, T. L. (1995). Characteristics of ego -and taskoriented students: A comparison of two methodologies. British Journal of Educational Psychology, 65, 125-138. 
Skaalvik, E. M. (1997). Self-enhancing and self-defeating ego orientation: Relations with task and avoidance orientation, achievement, self-perceptions, and anxiety. Journal of Educational Psychology, 89, 71-81.

Suárez, J. M., Cabanach, R. G. \& Valle, A. (2001). Multiple-goal pursuit and its relation to cognitive, self-regulatory, and motivational strategies. British Journal of Educational Psychology, 71, 561 - 572.

Valle, A., Cabanach, R. G., Núñez, J. C., GonzálezPienda, J. A., Rodríguez, S. \& Piñeiro, I. (2003). Multiple goals, motivation and academic learning. British Journal of Educational Psychology, 73, 71-87.

Valle, A., Núñez, J. C., Cabanach, R. G., Rodríguez, S., González-Pienda, J. A. \& Rosário, P. (2009). Perfiles motivacionales en estudiantes de secundaria: análisis diferencial en estrategias cognitivas, estrategias de autorregulación y rendimiento académico. Revista Mexicana de Psicología, 26, 113-124.

Valle, A., Núñez, J. C., Cabanach, R. G., Rodríguez, S., González-Pienda, J. A., Rosário, P., Muñoz-Cadavid, M. A. \& Cerezo, R. (2009). Academic goals and learning quality in higher education students. The Spanish Journal of Psychology, 12, 96-105.

Wentzel, K. R. (2000). What is it that I'm trying to achieve? Classroom goals from a content perspective. Contemporary Educational Psychology, 25, 105-115.

Wolters, C., Yu, S. \& Pintrich, P. R. (1996). The relation between goal orientation and students' motivational beliefs and self-regulated learning. Learning and Individual Differences, 8, 211-238. 
